\title{
Physical parameter determination of seven RR Lyrae stars in Bootes J. H. Peña, ${ }^{1}$ A. Arellano, ${ }^{1}$ J. P. Sareyan, ${ }^{2}$ R. Peña, ${ }^{1}$ M. Alvarez ${ }^{1}$ \\ ${ }^{1}$ Instituto de Astronomía, UNAM, México D.F., México \\ ${ }^{2}$ Observatoire de la Cote d'Azur, France
}

\begin{abstract}
uvby $\beta$ photoelectric photometry of the RR Lyrae stars AE, RS, ST, TV, TW, UU, and $\mathrm{XX}$ Bootis has been acquired in order to determine the physical parameters of the stars. We used the correlation between the Fourier parameters derived from the light curves and the physical parameters such as the absolute magnitude $M_{v}$, intrinsic colour $(B-V)_{0}$ and metal abundance $[\mathrm{Fe} / \mathrm{H}]$. Once reddening has been determined, unreddened indices are obtained and $T_{\text {eff }}$ and $\log g$ followed along the cycle, using a comparison with the theoretical models given for our determined metallicity.
\end{abstract}

\section{Analysis}

Our knowledge on stellar evolution comes from a direct comparison between the observational determination of the star's basic physical parameters (i.e. $T_{\text {eff }}, g$, luminosity, metallicity and eventual pulsation periods) and models. In the case of the RR Lyrae variables, some of the goals can be attained from the relationships that have been developed by Kovács \& Jurcsik in several papers (Jurcsik \& Kovács 1996, Kovács \& Jurcsik 1996, 1997, Jurcsik 1998). As Kovács \& Walker (2001) state in their review paper, this method is empirical and based on the assumption that the period and the shape of the light curve are directly correlated with the physical parameters. Since we have obtained simultaneous uvby $\beta$ photoelectric photometry, the precise variation of these parameters along the cycle can be determined for each star.

The observations were all taken at the Observatorio Astronómico Nacional, Mexico. The $1.5 \mathrm{~m}$ telescope, to which a spectrophotometer was attached, was used in all seasons. The physical parameters can be determined with the ad-hoc models. From the data of several photometric campaigns we obtained uvby $\beta$ photometry, which was used to calculate the physical parameters of each star. The fit with our observations has been considered as reasonable when the rms error is the same order of magnitude as the detected amplitude. Reddening has been estimated by considering different objects in the same direction. Once reddening has been fixed, the unreddened Strömgren indices give the variation along the cycle of the effective temperature and surface gravity. These results are summarized in Table 1 which reports the physical parameters. The last two columns list the number of data points and the standard deviation of the residuals.

Acknowledgments. This paper was partially supported by Papiit IN108106. This article has made use of the SIMBAD database operated at CDS, Strasbourg, France and ADS, NASA Astrophysics Data Systems hosted by Harvard-Smithsonian Center for Astrophysics. 
Table 1: Physical parameters from the calibrations for studied RR Lyrae stars

\begin{tabular}{|l|c|c|c|c|c|c|c|c|}
\hline ID & {$[F e / H]$} & $T_{\text {eff }}(K)$ & $M v$ & $\log L / L \odot$ & $\log P$ & Type & data points & $\sigma$ \\
\hline RS & -0.281 & 6889 & 1.00 & 1.48 & -.423 & AB & 43 & 0.057 \\
ST & -1.306 & 6444 & 0.71 & 1.62 & -.206 & AB & 39 & 0.088 \\
TW & -1.227 & 6569 & 0.82 & 1.58 & -.274 & AB & 33 & 0.124 \\
UU & -0.487 & 6795 & 0.88 & 1.54 & -.340 & AB & 25 & 0.084 \\
XX & -0.282 & 6649 & 0.89 & 1.53 & -.236 & AB & 34 & 0.055 \\
AE & -1.293 & 7370 & 0.61 & 1.68 & -.502 & $\mathrm{C}$ & 138 & 0.018 \\
TV & -1.877 & 7200 & 0.34 & 1.79 & -.505 & $\mathrm{C}$ & 500 & 0.014 \\
\hline
\end{tabular}

\section{References}

Jurcsik J., 1998, A\&A, 333, 571

Jurcsik J., Kovács G., 1996, A\&A, 312, 111

Kovács G., Jurcsik J., 1996, ApJ, 466, L17

Kovács G., Jurcsik J., 1997, A\&A, 322, 218

Kovács G., Walker A. R., 2001, A\&A, 371, 579

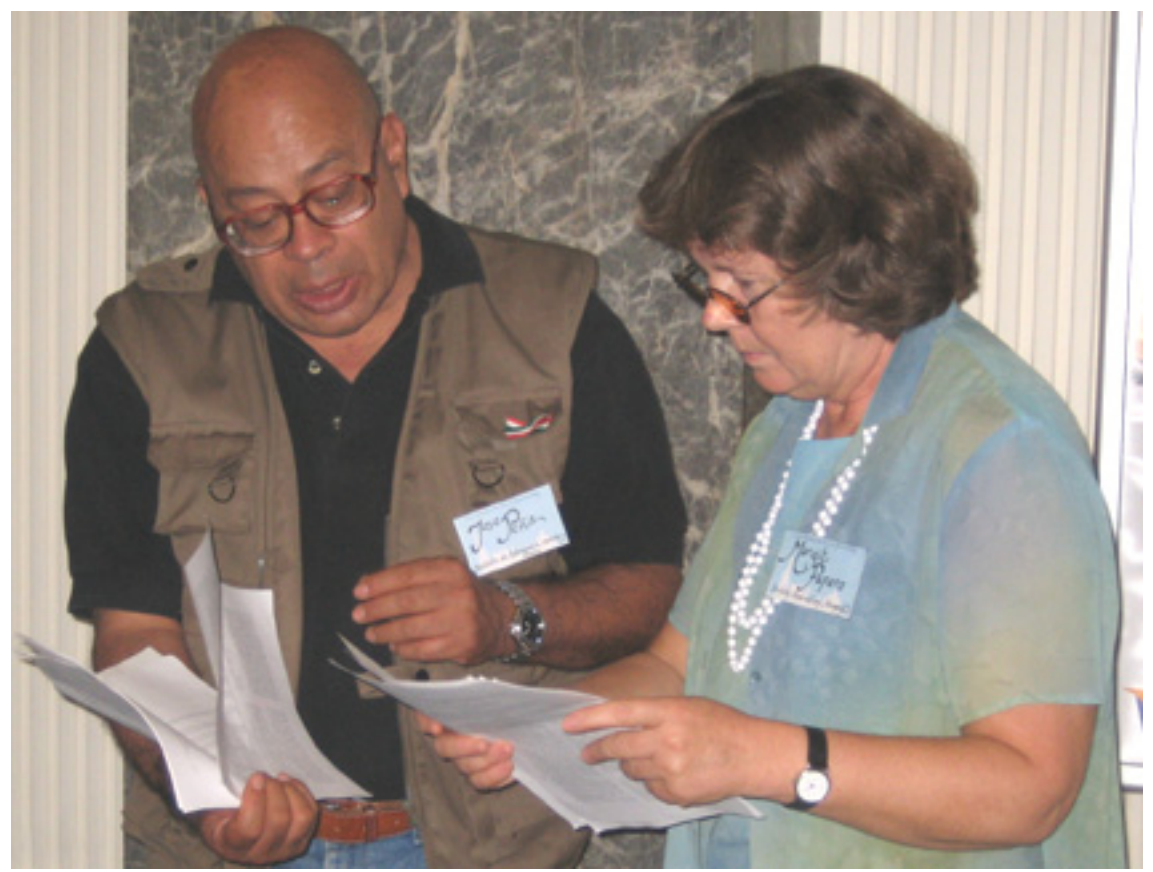

José Peña and Margit Paparó in intensive discussions. 